\title{
$k$-HYPONORMALITY OF WEIGHTED SHIFTS
}

\author{
SCOTT MCCULLOUGH AND VERN PAULSEN
}

(Communicated by Theodore W. Gamelin)

\begin{abstract}
An operator $T$ is defined to be $k$-hyponormal if the operator matrix $\left(\left[T^{* j}, T^{i}\right]\right)_{i, j=1}^{k}$ is positive, where $[A, B]=A B-B A$. In $A$ note on joint hyponormality, Proc. Amer. Math. Soc. 107 (1989), 187-195, we proved that $k$-hyponormality is equivalent to a Bram-type condition, namely, that the operator matrix $\left(T^{* j} T^{i}\right)_{i, j=0}^{k}$ is positive. In this note we prove that for weighted shifts, $k$-hyponormality is equivalent to an Embry-type condition, namely, that the operator matrix $\left(T^{* i+j} T^{i+j}\right)_{i, j=0}^{k}$ is positive. We give an example to show that this latter condition fails even for a rank one perturbation of a weighted shift. For weighted shifts this Embry condition reduces to the positivity of a sequence of $(k+1) \times(k+1)$ Hankel matrices and we use this reduction to give a new proof of one of the principal results of Curto.
\end{abstract}

\section{INTRODUCTION}

An operator $T$ is hyponormal if the commutator $\left[T^{*}, T\right]=T^{*} T-T T^{*}$ is positive and polynomially hyponormal if every polynomial in $T$ is hyponormal, i.e., if every finite linear combination of the powers of $T$ is hyponormal. An operator is subnormal if it is the restriction of a normal operator to an invariant subspace.

Every subnormal operator is polynomially hyponormal and an old problem in operator theory is to determine whether the converse is true. Recent research on this problem has led many authors $[1,4-6]$ to consider conditions that fall in between hyponormality and subnormality.

A result of Bram [2] asserts that an operator $T$ is subnormal if and only if the operator matrices $\left(\left[T^{* j}, T^{i}\right]\right)_{i, j=1}^{k}$ are positive for all $k$. An operator is $k$-hyponormal if the above operator matrix is positive for some fixed integer $k$. Thus, Bram's result is that $T$ is subnormal if and only if $T$ is $k$-hyponormal for every $k$. An equivalent definition of $k$-hyponormality is to require that the operator matrix $\left(T^{* j} T^{i}\right)_{i, j=0}^{k}$ be positive [9].

There is a second characterization of subnormality due to Embry [8]. Embry's condition states that an operator is subnormal if and only if the operator matrices $\left(T^{* i+j} T^{i+j}\right)_{i, j=0}^{k}$ are positive for every $k$. It is natural to expect some relation between the $(k+1) \times(k+1)$ Embry and Bram conditions. By pre- and

Received by the editors February 19, 1991.

1991 Mathematics Subject Classification. Primary 47B20; Secondary 47B37.

The second author's research was supported in part by the NSF. 
post-multiplying $\left(T^{* j} T^{i}\right)_{i, j=0}^{k}$ by a diagonal operator matrix whose diagonal entries are powers of $T$, it is easily seen that positivity of $\left(T^{* j} T^{i}\right)_{i, j=0}^{k}$ implies positivity of $\left(T^{* i+j} T^{i+j}\right)_{i, j=0}^{k}$. Moreover, if $T$ is invertible, then by using the inverse of this diagonal we see that the positivity of these two operator matrices is equivalent.

We give an example that shows positivity of these two operator matrices is not equivalent for noninvertible $T$. However, we prove that for weighted shifts the positivity of these two operator matrices is equivalent. Thus, $k$-hyponormality of a weighted shift is equivalent to the positivity of the $(k+1) \times(k+1)$ operator matrix $\left(T^{* i+j} T^{i+j}\right)_{i, j=0}^{k}$ each of whose entries is itself a (infinite) diagonal matrix. The positivity of such an operator reduces to the positivity of an infinite sequence of scalar $(k+1) \times(k+1)$ Hankel matrices. These facts allow us to give new proofs of some of Curto's results [6], which avoid the use of Constantinescu results [3].

There is a second reason that we believe this further simplification of the weighted shift case is important. In [9] we proved that if there exists a polynomially hyponormal operator that is not subnormal, then necessarily there exists a weighted shift with this property. Thus, it is sufficient to restrict our attention to weighted shifts in any analysis of this problem.

\section{The BRAM AND EMbry CONDITIONS}

As we noted in the introduction, positivity of $\left(T^{* j} T^{i}\right)_{i, j=0}^{k}$ always implies positivity of $\left(T^{* i+j} T^{i+j}\right)_{i, j=0}^{k}$ and the converse is true for invertible $T$. In this section we prove the converse is true for unilateral weighted shifts and give an example to show that it is not true in general. We then apply this Embry condition to give a fairly simple criteria for $k$-hyponormality of weighted shifts that we use to rederive an essential result of Curto, from which many of his other results follow. We begin with an example.

Example 2.1. Let $T=S+E_{22}$ where $S$ is the unilateral shift with weight sequence $\left\{a_{n}\right\}$ satisfying $a_{0}=1, a_{1}=\sqrt{2}$, and $a_{n}=2$ for all $n \geq 2$ and where $E_{22}$ is the matrix with 1 in the $(2,2)$ entry and 0 elsewhere. We shall prove that $\left(\begin{array}{ll}I & T^{*} \\ T & T^{*} T\end{array}\right)$ is not positive, but that $\left(\begin{array}{cc}I & T^{*} T \\ T^{*} T & T^{* 2} T^{2}\end{array}\right)$ is positive.

To see that the first operator matrix is not positive, we first need to compute $T^{*} T$. We find that $T^{*} T$ is the direct sum of the $2 \times 2$ matrix $\left(\begin{array}{ll}1 & 1 \\ 1 & 3\end{array}\right)$ with a diagonal matrix all of whose diagonal entries are 4.

Thus if we compress $\left(\begin{array}{ll}I & T^{*} \\ T & T^{*} T\end{array}\right)$ to the six-dimensional space spanned by the first three basis vectors in each copy of the space, i.e., the linear span of $e_{1} \oplus 0$, $e_{2} \oplus 0, e_{3} \oplus 0$ and $0 \oplus e_{1}, 0 \oplus e_{2}, 0 \oplus e_{3}$, we obtain the matrix

$$
\left(\begin{array}{cccccc}
1 & 0 & 0 & 0 & 1 & 0 \\
0 & 1 & 0 & 0 & 1 & \sqrt{2} \\
0 & 0 & 1 & 0 & 0 & 0 \\
0 & 0 & 0 & 1 & 1 & 0 \\
1 & 1 & 0 & 1 & 3 & 0 \\
0 & \sqrt{2} & 0 & 0 & 0 & 4
\end{array}\right) .
$$

A straightforward computation shows that this matrix has determinant -2 and so is not positive. 
We must now show that the second operator matrix is positive. For this verification we compute $T^{* 2} T^{2}$, which we find is the direct sum of the $2 \times 2$ matrix $\left(\begin{array}{ll}3 & 3 \\ 3 & 11\end{array}\right)$ with a diagonal matrix all of whose diagonal entries are 16 . Thus, to check the positivity of

$$
\left(\begin{array}{cc}
I & T^{*} T \\
T^{*} T & T^{* 2} T^{2}
\end{array}\right)
$$

it suffices to verify that the $4 \times 4$ matrix

$$
\left(\begin{array}{cccc}
1 & 0 & 1 & 1 \\
0 & 1 & 1 & 3 \\
1 & 1 & 3 & 3 \\
1 & 3 & 3 & 11
\end{array}\right)
$$

and the $2 \times 2$ matrix $\left(\begin{array}{cc}1 & 4 \\ 4 & 16\end{array}\right)$ are positive.

The $2 \times 2$ matrix is easily seen to be positive. The $4 \times 4$ matrix is also positive since it is equal to $U^{*} U$ where

$$
U=\left(\begin{array}{cccc}
1 & 0 & 1 & 1 \\
0 & 1 & 1 & 3 \\
0 & 0 & 1 & -1
\end{array}\right)
$$

This final computation shows that the example has the advertised properties.

We are now prepared to prove our main theorem.

Theorem 2.2. Let $T$ be a unilateral weighted shift with nonzero weight sequence. Then, for each $k$, the operator matrix $\left(T^{* j} T^{i}\right)_{i, j=0}^{k}$ is positive if and only if $\left(T^{* i+j} T^{i+j}\right)_{i, j=0}^{k}$ is positive.

Proof. From our earlier remarks, it will be sufficient to prove that positivity of the latter matrix implies positivity of the former matrix. We regard the weighted shift as acting on the Hilbert space $l_{2}$, so that each of the operator matrices acts upon $H=l_{2}^{k+1}$. Let $\left\{e_{n}\right\}_{n=1}^{\infty}$ be the canonical orthonormal basis for $l_{2}$ so that $T e_{n}=a_{n} e_{n+1}$ for some sequence of scalars $\left\{a_{n}\right\}$. Set $A=\left(T^{* j} T^{i}\right)_{i, j=0}^{k}$, $B=\left(T^{* i+j} T^{i+j}\right)_{i, j=0}^{k}$. Let $\mathscr{M}=l_{2} \oplus T l_{2} \oplus \cdots \oplus T^{k} l_{2}$, so that for $m=h_{0} \oplus T h_{1} \oplus$ $\cdots \oplus T^{k} h_{k}$ in $\mathscr{M}$ we have $\langle A m, m\rangle=\sum_{i, j=1}^{k}\left\langle T^{* j} T^{i} T^{j} h_{j}, T^{i} h_{i}\right\rangle=\langle B h, h\rangle$ where $h=h_{0} \oplus h_{1} \oplus \cdots \oplus h_{k}$. By hypothesis $\langle B h, h\rangle \geq 0$, and hence the compression of $A$ to $\mathscr{M}$ is positive. Since $A$ is selfadjoint and $\mathscr{M}$ is invariant for $A, \mathscr{M}$ reduces $A$. Therefore, the proof will be complete once we show that the compression of $A$ to the finite-dimensional space $\mathscr{M}^{\perp}$ is also positive.

To this end note that $\mathscr{M}^{\perp}=0 \oplus \mathscr{L}_{1} \oplus \cdots \oplus \mathscr{L}_{k}$ where $\mathscr{L}_{j}$ is the span of $\left\{e_{n}\right\}_{n=1}^{j}$. Let $e_{j n}=0 \oplus \cdots \oplus 0 \oplus e_{n} \oplus 0 \oplus \cdots \oplus 0$, where the $e_{n}$ is in the $j$ th space. Thus $e_{j n}, 1 \leq n, j \leq k$, is an orthonormal basis of $\mathscr{M}^{\perp}$. For convenience in notation, we set $e_{j n}=0$ for $n \leq 0$. We let $\mathscr{K}_{l}$ denote the span of $e_{l, 1}, e_{l, 2}, \ldots, e_{l, k+1-l}$ for $l=2, \ldots, k$. Thus $e_{j, n}$ is in $\mathscr{K}_{l}$ if and only if $j-n=l-1$. It is easily seen that $\mathscr{K}_{i} \perp \mathscr{K}_{j}$ for $i \neq j$ and that $\mathscr{M}^{\perp}=\mathscr{K}_{2} \oplus \cdots \oplus \mathscr{K}_{k}$. Note also that since $T^{* j} T^{i} e_{j, n}$ is a multiple of $e_{i, n+i-j}$ and $j-n=i-(n+i-j)$ we have each space $\mathscr{K}_{l}$ is invariant for $A$, and hence reduces $A$. 
Finally, note that each vector $h$ in $\mathscr{K}_{l}$ has the form $0 \oplus \cdots \oplus 0 \oplus c_{l+1} e_{1} \oplus$ $\cdots \oplus c_{k} T^{k-l} e_{1}$ for some scalars $c_{l}, \ldots, c_{k}$. Thus

$$
\begin{aligned}
\langle A h, h\rangle & =\sum_{i, j=l}^{k}\left\langle T^{* j} T^{i} c_{j} T^{j-l} e_{1}, c_{i} T^{j-l} e_{1}\right\rangle \\
& =\sum_{i, j=1}^{k} c_{j} \overline{c_{i}}\left\langle T^{*(j+i-l)} T^{j+i-l} e_{1}, e_{1}\right\rangle \\
& =\sum_{m, n=0}^{k-l} c_{m+2} \overline{c_{n+l}}\left\langle T^{*(m+n)} T^{m+n} e_{1}, e_{1}\right\rangle .
\end{aligned}
$$

This last term is seen to be positive since it is $\left\langle B h_{1}, h_{1}\right\rangle$ where $h_{1}=c_{l} e_{1} \oplus \cdots \oplus$ $c_{k} e_{1} \oplus 0 \oplus \cdots \oplus 0$.

Thus $A$ compressed to each space $\mathscr{K}_{l}$ is positive from which the result follows.

Using the above theorem it is now easy to obtain a result of Curto's.

Corollary 2.3 (Curto [6, Theorem 4]). Let $T$ be a unilateral weighted shift with weight sequence $\left\{a_{n}\right\}_{n=1}^{\infty}$ and let $b_{n}=\left|a_{1} \cdots a_{n}\right|^{2}$ with $b_{0}=1$. Then $T$ is $k$-hyponormal if and only if each of the $(k+1) \times(k+1)$ Hankel matrices $\left(b_{i+j+n}\right)_{i, j=0}^{k}$ is positive for every $n \geq 0$.

Proof. Note that $T^{* i+j} T^{i+j}$ is a diagonal operator whose $n$th diagonal entry is $b_{i+j+n} / b_{n}$. Thus the operator matrix $\left(T^{* i+j} T^{i+j}\right)_{i, j=0}^{k}$ is positive if and only if the scalar matrix $\left(b_{i+j+n}\right)_{i, j=0}^{k}$ is positive for every $n$.

As a final remark, we note that, following the proof of Theorem 2.2 , it is easily seen that if $T$ is an operator with $\left(T^{* i+j} T^{i+j}\right)_{i, j=0}^{k}$ positive, then $\left(T^{* j} T^{i}\right)_{i, j=0}^{k}$ restricted to the subspace $\mathscr{M}=0 \oplus R(T) \oplus \cdots \oplus R\left(T^{k}\right)$, where $R(S)$ denotes the closure of the range of $S$, is positive. Moreover, $\mathscr{M}$ is invariant for $\left(T^{* j} T^{i}\right)_{i, j=0}^{k}$ and hence reducing, so that the positivity or nonpositivity of $\left(T^{* j} T^{i}\right)_{i, j=0}^{k}$ is determined by its behavior on $\mathscr{M}^{\perp}$.

\section{REFERENCES}

1. A. Athavale, On joint hyponormality of operators, Proc. Amer. Math. Soc. (to appear).

2. J. Bram, Subnormal operators, Duke Math. J. 22 (1955), 75-94.

3. T. Constantinescu, Schur analysis of positive block-matrices, Operator Theory: Adv. Appl. 18 (1986), 191-206.

4. J. Conway and W. Szymanski, Linear combinations of hyponormal operators, Rocky Mountain J. Math. (to appear).

5. R. E. Curto, Joint hyponormality: a bridge between hyponormality and subnormality.

6. __ Quadratically hyponormal weighted shifts, Integral Equations Operator Theory (to appear).

7. R. E. Curto, P. Muhly, and J. Xia, Hyponormal pairs of commuting operators, Operator Theory: Adv. Appl. 35 (1988). 
8. M. Embry, A generalization of the Halmos-Bram condition for subnormality, Acta. Sci. Math. (Szeged) 35 (1973), 61-64.

9. S. McCullough and V. I. Paulsen, A note on joint hyponormality, Proc. Amer. Math. Soc. 107 (1989), 187-195.

Department of Mathematics, University of Florida, Gainesville, Florida 32611

Department of Mathematics, University of Houston, Houston, TeXas 77004 